\title{
Effects of imperfect quality items in the asymmetric information structure in supply chain model
}

\author{
Rita Yadav ${ }^{a}$, Sarla Pareek ${ }^{\mathrm{b}}$, Mandeep Mittal ${ }^{\mathrm{c}}$ and Sumil Mehta
}

\section{H R O N I C L E} \\ Article history: \\ Received March 2, 2017 \\ Received in revised format \\ October 10, 2017 \\ Accepted November 32017 \\ Available online \\ November 32017 \\ Keywords: \\ Supply chain \\ Imperfect quality items \\ Game theory \\ Non-cooperative games \\ Seller Stackelberg game \\ Symmetric and asymmetric \\ information structure
}

${ }^{a}$ Department of Applied Sciences and Humanities, Dronacharya College of Engineering, Gurugram, India

${ }^{b}$ Department of Mathematics and Studies, Banasthli Vidyapeeth, Jaipur, India

${ }^{c}$ Department of Computer Science and Engineering, Amity School of Engineering and Technology, New Delhi, India

\begin{abstract}
A B S T R A C T
Most of the supply chain models have been developed under symmetric information structure i.e. players have complete knowledge of each other's policies. But in most of the cases, players do not have complete information about the other players i.e. some information regarding their businesses is hidden. This paper studies supply chain model of imperfect quality items under asymmetric information in which unit price taken by the buyer and unit marketing expenditure are influencing product's demand. This information is hidden to seller. The seller delivers the supply to the buyer. Each delivered lot goes through an inspection process at the buyer's side. In the inspection process, the items are divided into two categories. The first category is perfect quality items while the second category is of imperfect quality items. After the inspecting the lots, the perfect quality commodities are to be sold at selling price and the imperfect items are supposed to get sold at a discounted price. The interaction between two constituents of the supply chain is modeled by non-cooperative Seller Stackelberg game approach in which the role of leader is played by the seller while the role of follower is played by the buyer. In our proposed model, the seller does not have information related to market demand but the buyer does. Numerical examples and sensitivity analysis explain the significance of the theory.
\end{abstract}

\section{Introduction}

Game theory is the study and analysis of mathematical models of cooperation and human conflict in the presence of a competition. For some contexts, game theory is about the science of strategies, or at least the optimized decision-taking approach of independent and competitive actors in a strategic setting. The key role in game theory was played by mathematicians John Nash and John von Neumann, as well as economist Oskar Morgenstern. Various researches have been published related to supplychain management. Sarmah et al. (2006) developed a buyer seller coordination models in supply chain channel. Different mechanisms have been brought related to supply chain like credit option, quantity discount (Chiang et al., 1994; Weng, 1995), buy back and quantity flexibility. Cachon and Lariviere (2005) explained the contract of sharing the revenue and compared this contract to various other supply chain contracts: sales-rebate contracts buy-back contracts, quantity-flexibility contracts, price-discount contracts, franchise contracts, and quantity discounts.

* Corresponding author

E-mail address: mittal mandeep@yahoo.com (M. Mittal)

C) 2018 Growing Science Ltd. All rights reserved.

doi: $10.5267 /$ j.uscm.2017.11.002 
Under the fixed demand, several researches (Sucky, 2005a, 2006b; Heuvel et al., 2007; Chan \& Kingsman, 2007; Dai \& Qi, 2007; Papachristos \& Konstantaras, 2006) have contributed in the field of supply chain in which they determined the optimal lot size and order cycle to achieve maximum profit of supply chain channel. Initially, Schwaller (1988) explored an EOQ model on defective items under inspection cost. Salameh and Jaber (2000) extended the E.O.Q. /E.P.Q. model for imperfect items and these imperfect items are to be sold at some discounted prices in the completion of inspection process in a single lot. By adding some constant parameter, Cárdenas-Barrón (2000) corrected the optimum order quantity formula obtained by Salameh and Jaber (2000). Goyal and Cárdenas-Barrón (2002) presented a simple way for EOQ model with items of imperfect quality and compared the result with Salameh and Jaber (2000) and obtained optimal results. Maddah and Jaber (2008) extended their work by analyzing the effect of screening speed and supply process' variability on the lot size. They illustrated in their model that when the divergence of the yield rate is lower, then the order quantity is higher as compared to the one in classical EOQ.

Fixed demand is avoided by many researchers in supply chain channel in which the optimal size of the lot and optimal price are determined to maximize its profit. Researchers like Lee (1993), Abad (1994), Lee et al. (1996), Kim and Lee (1998), Jung and Klein (2001a, 2005b) showed their interest to establish supply chain model to calculate the optimal policy for buyer and seller, in which final demand is price sensitive. With the same demand function Jaggi et al. (2011) constructed a model which maximizes the retailer's total profit and jointly optimize the price and number of replenishments.

Some another researchers (e.g. Freeland, 1980; Lee and Kim, 1993; Sadjadi et al., 2005) proposed models in which demand depends not only on selling price but also on marketing expenditure. Abad (1994) work was extended by Abad and Jaggi (2003), where seller presents credit to the buyer when the demand is price sensitive. In this case, seller and buyer interact with each other and their profits are maximized with optimal policies under non-cooperative and cooperative game theoretic approach. Esmaeili et al. (2009) also established cooperative and non-cooperative game supply-chain model in which demand depends on marketing expenditure and price.

In the above papers, buyer and seller have all the information about each other's activities. In many situation, some private information related to the business is hidden to other players. Harsanyi (1967, $1968 \mathrm{a}, \mathrm{b})$ developed the theory about analysis of highly innovative games of incomplete information called Bayesian games. Corbett and Groote (2000) derived the optimal quantity discount policy using asymmetric information and compared the results with the conditions, where the seller has full information. Lau and Lau (2005) presented an illustration of the introduction about informationasymmetry and stochasticity on 2-echelon supply chain gaming models and demonstrated its necessity and practicality of using it rather than the usual deterministic symmetric information way in various supply-chain models.

Lau et al. (2007) demonstrated a procedure using stochastic and asymmetric demand information for a superior manufacturer to create a profit-maximizing volume-discount scheme. Sucky (2006) presented a bargaining model regarding the buyer-cost structure considering that the buyer possesses the authority to impose its individual optimal policy under asymmetric information. Reyes (2005) extended the newsboy problem for buyer and seller in the asymmetric market information structure in which market demand is familiar to the buyer and presented the model in which efficiency of supply chain channel is improved by taking the advantage of the buyer by the seller. Esmaeiliand Zeephongsekul (2010) extended his work on asymmetric-information game by using the same demand function. Zhang and Panlop (2013) proposed a supply chain model with credit option under symmetric as well as asymmetric information and also draw some conclusion by taking different probability density function.

Every production system may produce some defective items. Jaggi et al. (2013) suggested that defectives can be detected with the help of inspection process after the delivery which can impact the ordered quantity. Thus, inspection of the items cannot be ignored at the buyer's end. None of the authors worked on the imperfect quality items in supply chain models with symmetric and asymmetric information structure. In this paper, we assume that the rate of production of the seller is linearly 
associated to demand rate. Also, the demand is sensitive to marketing expenditure and selling price. The seller and buyer interact with each other and this interaction is shown by several supply chain models with imperfect items under non-cooperative game with both asymmetric and symmetric information structure. Seller Stackelberg is illustrated under non-cooperative approach. In this approach, seller plays as leader and the buyer plays as follower. Numerical examples show that profit function of the symmetric case is more in comparison to the profit function obtained in the asymmetric case. We assume the seller's belief on the buyer's type follow uniform distribution. First section consists of Introduction and Literature review. Second section introduces notation and assumptions used in the paper. In third section, we summarize buyer's model, seller's model and primary concepts of asymmetric information game. Fourth section deals with the non-cooperative seller Stackelberg game in both asymmetric and symmetric information scenario. In fifth and six sections, numerical examples and sensitivity analysis are given. In the last two sections, observations and conclusion are provided.

\section{Notations and assumptions}

\subsection{Notations}

The notations used throughout the paper.

\section{Decision variables}

$c_{b} \quad$ Seller's selling price (decision variable of seller) (\$/unit)

$Q \quad$ Order quantity determined by the seller (decision variable of seller) (units)

$M \quad$ Marketing expenditure cost (decision variable of buyer) (\$/unit)

$p_{b} \quad$ Buyer's retail price (decision variable of buyer) (\$/unit)

\section{Parameters}

$A_{b} \quad$ Buyer's order placement cost (\$/order)

$A_{s} \quad$ Ordering cost of seller (\$/order).

$H_{b} \quad$ Inventory carrying cost

C Seller's unit purchasing cost (\$/unit)

I Percentage of inventory holding cost per unit per year.

$T_{1} \quad$ Cycle time in years for the buyer, $T_{1}=Q(1-\alpha) / D$

$T_{2}$ Cycle time in years for the seller, $T_{2}=Q / D$

$T \quad$ Cycle time in years for the seller Stackelberg model, $T=\operatorname{Max}\left(T_{1}, T_{2}\right)$

$\alpha \quad$ Percentage of defective items delivered to buyer by the seller

$\lambda$ Buyer's screening rate (unit/year)

$c_{S} \quad$ Defective quality items' cost per unit (\$/year)

$t \quad$ Required time to screen the defective items, $t=Q / \lambda$ (Years)

$k$ Demand function's scaling constant $(k>0)$

$r$ Production rate(units/day)

$u \quad$ Production function's scaling constant $(u \geq 1)$

$e \quad$ Demand function's price elasticity $(e>1)$

$\beta$ Marketing expenditure elasticity of demand function $(0<\beta<1, \beta+1<e)$ 
$D$ Annual demand rate (unit/year), Demand is presumed as function of selling price $p_{b}$ and marketing expenditure $\mathrm{M}$ i.e. $D=k p_{b}{ }^{-e} M^{\beta}$

\subsection{Assumptions}

1. The annual demand is sensitive to marketing expenditure and selling price. (Esmaeili et al., 2009), which is known to the buyer through the parameters $e, \beta$ but uknown to the seller.

2. Planning horizon is infinite.

3. Every lot has $\alpha$ percentage of defectives items of known probability density function.

4. Shortages are not permitted. We assume the production rate $r$ and demand rate $d$ is linearly related defined as $r=u d, \quad u \geq 1$ (Esmaeili et al., 2009)

5. Generally, in supply chain model, ordered quantity is determined by the buyer but we assume order quantity or lot size is the decision variable of the seller.

\section{Mathematical models}

\subsection{Buyer's model}

The objective of the buyer is to find the retailer price, $p_{b}$, and marketing expenditure, $M$, such that the net profit gets maximized. In this paper, the demand relies on the marketing expenditure and selling price. We consider a supply chain model with imperfect quality items. The seller supplies the items to the buyer with unit price. After delivery of $Q$ units (assumed), the lot goes through an inspection process at the buyer's end. Defective items and non-defective items are separated through the inspection process from the entire delivered quantity $Q$ at the rate of $\lambda$ units per unit time. We assumed that, in a lot of $Q$ quantity items, $\alpha$ percent items are found defectives with known probability density function. Defective items $\alpha Q$ are sold in a single lot at the end of screening time, $t=Q / \lambda$ at a discounted price $c_{S}$ and nondefective items, $(1-\alpha) Q$ are sold at a price $p_{b}$. Thus the total revenue of the buyer is $p_{b}(1-\alpha) Q+$ $c_{s} \alpha Q$ in which defective items $\alpha Q$ are sold at a discounted price $c_{s}$ and non-defective items, $(1-\alpha) Q$ are sold at a selling price $p_{b}$. The purchasing cost of the buyer of $Q$ quantity at a price $c_{b}$ is $c_{b} Q$. The marketing cost of the buyer of $Q$ quantity is $M Q$ and ordering cost of the buyer is $A_{b}$, and inventory carrying cost will be equal to $H_{b}\left(\frac{Q(1-\alpha) T}{2}+\frac{\alpha Q^{2}}{\lambda}\right)$, where $H_{b}=I c_{b}$.

Total annual profit for the buyer is denoted by $\mathrm{TP}_{b}\left(p_{b}, M\right)$ and is given by

$\mathrm{T} P_{b}\left(p_{b}, M\right)=$ Sales Revenue - Purchasing cost - Marketing cost- Ordering cost - Inventory carrying

$$
\operatorname{cost}=p_{b}(1-\alpha) Q+c_{s} \alpha Q-c_{b} Q-M Q-A_{b}-\left(\frac{Q(1-\alpha) T_{1}}{2}+\frac{\alpha Q^{2}}{\lambda}\right) H_{b}
$$

$\mathrm{Put}_{1}=\frac{(1-\alpha) Q}{D}, t=\frac{Q}{\lambda}, H_{b}=\mathrm{Ic}_{b}$ then buyer's profit becomes

$$
\begin{aligned}
T P_{b}\left(p_{b}, M\right)= & p_{b}(1-\alpha) Q+c_{s} \alpha Q-c_{b} Q-M Q-A_{b}-\left(\frac{Q^{2}(1-\alpha)^{2}}{2 D}+\frac{\alpha Q^{2}}{\lambda}\right) \mathrm{I} c_{b} \\
& =p_{b}(1-\alpha) Q+c_{s} \alpha Q-c_{b} Q-M Q-A_{b}-\left(\frac{Q^{2}(1-\alpha)^{2}}{2 D}+\frac{\alpha Q^{2}}{\lambda}\right) \mathrm{I} c_{b}
\end{aligned}
$$

Thus, the total expected buyer's profit is given by

$$
\begin{aligned}
E\left[T P_{b}\left(p_{b}, M\right)\right]= & p_{b} E[1-\alpha] Q+c_{s} E[\alpha] Q-c_{b} Q-M Q-A_{b}-\left(\frac{Q^{2}(1-\alpha)^{2}}{2 D}+\frac{\alpha Q^{2}}{\lambda}\right) \mathrm{I} c_{b} \\
& =p_{b}(1-E[\alpha]) Q+c_{S} E[\alpha] Q-c_{b} Q-M Q-A_{b}-\left(\frac{Q^{2} E\left[(1-\alpha)^{2}\right]}{2 D}+\frac{E[\alpha] Q^{2}}{\lambda}\right) I c_{b}
\end{aligned}
$$


By using, Renewal theory as used in Maddah and Jaber (2008), we have the expected total profit per cycle for the buyer,

$$
\begin{aligned}
& E\left[\operatorname{TP}_{b}^{c}\left(p_{b}, M\right)\right]=\frac{E\left[T P_{b}\left(p_{b}, M\right)\right]}{E\left[T_{1}\right]}=\frac{D}{Q(1-E[\alpha])} E\left[T P_{b}\left(p_{b}, Q\right)\right] \\
& =\frac{D}{Q(1-E[\alpha])}\left[p_{b}(1-E[\alpha]) Q+c_{S} E[\alpha] Q-c_{b} Q-M Q-A_{b}-\left(\frac{Q^{2} E\left[(1-\alpha)^{2}\right]}{2 D}+\frac{E[\alpha] Q^{2}}{\lambda}\right) I c_{b}\right] \\
& =p_{b} D+\frac{1}{(1-E[\alpha])}\left[c_{S} E[\alpha] D-c_{b} D-M D-\frac{A_{b} D}{Q}-\left(\frac{Q E\left[(1-\alpha)^{2}\right]}{2}+\frac{E[\alpha] Q D}{\lambda}\right) I c_{b}\right]
\end{aligned}
$$

In this paper, $D$, demand function assumed as, $D=K p_{b}{ }^{-e} M^{\beta}$.

$$
E\left[T P_{b}^{c}\left(p_{b}, M\right)\right]=\mathrm{K}_{b}{ }^{-e+1} M^{\beta}+\frac{\mathrm{Kp}_{b}{ }^{-e} M^{\beta}}{(1-E[\alpha])}\left[c_{s} E[\alpha]-c_{b}-M-\frac{A_{b}}{Q}-\frac{E[\alpha] Q}{\lambda} I c_{b}\right]-\left(\frac{Q E\left[(1-\alpha)^{2}\right]}{2(1-E[\alpha])} I c_{b}\right)
$$

The aim is to find the optimal value of $p_{b}$ and $M$ which will maximize the expected profit of buyer. The necessary and sufficient condition for expected total profit per cycle must be satisfied. The optimal value of $p_{b}$ can be obtained by differentiating Eq. (1) with respect to $p_{b}$ for fixed $M$ and setting it to zero,

$$
\begin{aligned}
& \frac{\partial E\left[T P^{c}\left(p_{b}, M\right)\right]}{\partial p_{b}}=0, \text { yields, } \\
& p_{b}=\frac{e}{(e-1)(1-E[\alpha])}\left[M+c_{b}+\frac{A_{b}}{Q}+\frac{I c_{b} E[\alpha] Q}{\lambda}-c_{s} E[\alpha]\right.
\end{aligned}
$$

Given expected profit function is strictly pseudo concave (Bazaraa et al., 2013) with respect to $p_{b}$ for fixed $M$ substituting the value of Eq. (2) into Eq. (1), we get,

$$
\begin{aligned}
E\left[T P_{b}^{c}\left(p_{b}(M), M\right)\right] & \\
& =\frac{K}{e}\left[\frac{e}{(e-1)(1-E[\alpha])}\left[M+c_{b}+\frac{A_{b}}{Q}+\frac{I c_{b} E[\alpha] Q}{\lambda}-c_{s} E[\alpha]\right]^{-e+1} M^{\beta}\right. \\
& -\left(\frac{Q E(1-\alpha)^{2}}{2(1-E[\alpha])} I c_{b}\right)
\end{aligned}
$$

First order condition with respect to $M$ which maximize the buyer's expected profit function i.e. $\frac{\partial E\left[T P^{c}\left(p_{b}(M), M\right)\right]}{\partial M}=0$, gives the result

$$
M=\frac{\beta}{(e-\beta-1)}\left[c_{b}+\frac{A_{b}}{Q}+I c_{b} E[\alpha] Q-c_{S} E[\alpha]\right],(\beta+1<e)
$$

Sufficient condition $\frac{\partial^{2} E\left[T P^{c} b\left(p_{b}(M), M\right)\right]}{\partial M^{2}}<0$ is also satisfied i.e. given Expected profit function of the buyer is concave with respect toM. Substituting Eq. (4) into Eq. (2), we have

$$
p_{b}=\frac{e}{(e-\beta-1)(1-E[\alpha])}\left[c_{b}+\frac{A_{b}}{Q}+I c_{b} E[\alpha] Q-c_{S} E[\alpha]\right], \beta+1<e
$$


Concavity of expected total profit $E\left[T P^{c}{ }_{b}\left(p_{b}, M\right)\right]$ in Eq. (1) with respect to $p_{b}$ and $M$ is shown with the help of graph (Fig. 1)

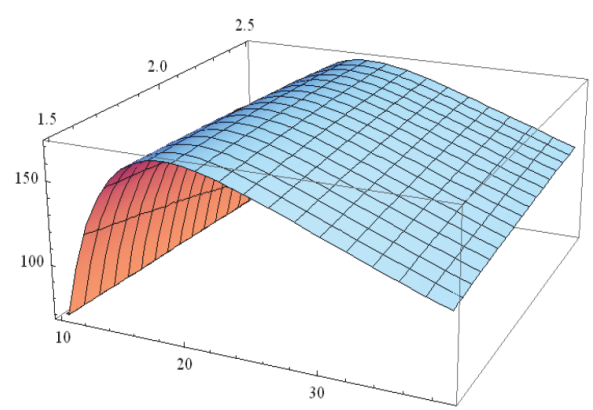

Fig. 1. Optimal buyer's total expected profit with respect to $p_{b}$ and $M$

Since,

$$
\begin{aligned}
& \frac{\partial^{2} E\left[T P^{c}{ }_{b}\left(p_{b}, M\right)\right]}{\partial p_{b}{ }^{2}}>0, \frac{\partial^{2} E\left[T P^{c} b_{b}\left(p_{b}, M\right)\right]}{\partial M^{2}}>0 \text { and } \\
& {\left[\frac{\partial^{2} E\left[T P^{c}{ }_{b}\left(p_{b}, M\right)\right]}{\partial p_{b}{ }^{2}}\right]\left[\frac{\partial^{2} E\left[T P^{c}{ }_{b}\left(p_{b}, M\right)\right]}{\partial M^{2}}\right]-\left[\frac{\partial^{2} E\left[T P^{c}{ }_{b}\left(p_{b}, M\right)\right]}{\partial p_{b} \partial M}\right]^{2}<0 }
\end{aligned}
$$

\subsection{Seller's model}

The seller delivers a lot to the buyer. The aim of the seller is to find the optimal selling price, $c_{b}$ and order quantity, $Q$ to maximize his net profit. The sales revenue generated by seller is $c_{b} Q$, purchasing cost per year is $c Q$, ordering cost per year is $A_{s}$ and holding cost of the seller is $\frac{1}{2} \operatorname{ICQT} \frac{D}{r}$. The profit function of the seller is denoted by $\operatorname{TP}_{s}\left(c_{b}, Q\right)$ and given by,

Seller's profit $=$ Sales Revenue - Purchasing Cost - Ordering cost - Holding cost

$$
\operatorname{Tp}_{s}\left(c_{b}, Q\right)=\left(c_{b} Q-C Q-A_{s}\right)-\frac{1}{2} I_{C Q T} \frac{D}{r}
$$

Cycle length, $T_{2}=\frac{Q}{D}$

Expected profit of seller per cycle is,

$$
\begin{aligned}
& E\left[\operatorname{TP}_{s}^{c}\left(c_{b}, Q\right)\right]=\frac{D}{Q}\left(c_{b} Q-C Q-A_{s}\right)-\frac{1}{2} I C Q u^{-1}=D\left(c_{b}-C-\frac{A_{s}}{Q}\right)-\frac{1}{2} I C Q u^{-1}, u= \\
& \frac{r}{D}
\end{aligned}
$$

The aim is to calculate the optimal value of $c_{b}$ and $Q$ which maximize the seller's expected profit. The optimal value of $Q$ can be obtained by differentiating Eq. (7) with respect to $Q$ for fixed $c_{b}$ and setting to zero, $\frac{\partial E\left[\mathrm{TP}_{S}^{c}\left(c_{b}, Q\right)\right]}{\partial Q}=0$, gives

$$
Q=\sqrt{\frac{2 A_{s} D}{I C u^{-1}}}
$$


The expected profit of the seller $E\left[\operatorname{TP}_{s}^{c}\left(c_{b}, Q\right)\right]$ function is concave in $Q$ because second order condition is,

$$
\frac{\partial^{2} E\left[\mathrm{TP}_{S}^{c}\left(c_{b}, Q\right)\right]}{\partial Q^{2}}=-2 \frac{A_{s} D}{Q^{3}}<0
$$

Substituting the value of $Q$ in the Eq. (7), we get

$$
E\left[\operatorname{TP}_{s}^{c}\left(c_{b}, Q\right)\right]=D\left[c_{b}-C-\frac{A_{s}}{\sqrt{\frac{2 A_{s} D}{I C u^{-1}}}}\right]-\frac{1}{2} I C u^{-1} \sqrt{\frac{2 A_{s} D}{I C u^{-1}}}
$$

Solving $E\left[\operatorname{TP}_{s}^{c}\left(c_{b}, Q\right)\right]=0$ for zero profit gives,

$$
c_{b_{0}}=C+\frac{A_{s}}{Q}+\frac{1}{2} I C(u D)^{-1} Q
$$

Above profit function is increasing linear function in $c_{b}$, the optimal value can be obtained by setting the highest price value through negotiation by the seller to the buyer. Therefore,

$$
\boldsymbol{c}_{\boldsymbol{b}}{ }^{*}=F c_{b_{0}}=F\left(C+\frac{A_{s}}{Q}+\frac{1}{2} I C(u D)^{-1} Q\right) \text { for some suitable, } F>1
$$

\subsection{Asymmetric/incomplete information game}

Asymmetric information game is a game in which at least one player possesses some private information regarding the game but other players may not know that information. Just before making a move in the game, a player has some initial private information, called the type of the player. For example, in an auction theory considering a single individual item, each player would know a valuation for the items deterministically, while the other players may have only probabilistic information about how much this player value the items. Strategic game with incomplete information based on the works described in Harsanyi $(1967,1968)$ can be defined in seller buyer supply chain model as follows.

$N=\left\{P_{1}, P_{2}\right\}$ is the set of player. In our model, buyer and seller are two players which are represented by $P_{1}$ and $P_{2}$. Since unit price is taken by the buyer and unit marketing expenditure influences the demand, $D=K p_{b}{ }^{-e} M^{\beta}$ of the product and buyer has better knowledge about the demand bur seller is not aware about the price elasticity, $e$ and marketing expenditure, $\beta$ which determine the market demand. In this model, all other input parameters are assumed to be known to both the players.

Let $\theta_{1}$ be the type of buyer. Therefore, based on our assumption,

$$
\theta_{1}=\{(e, \beta) ; \beta=L e, 0<\beta<1\}
$$

We denote a typical member of $\theta_{1}$ by $\mu_{1}$

$A=\left\{A_{1}, A_{2}\right\}$ is the set of actions of two player. These are also the decision variables of the players.

$$
A_{1}=\left\{p_{b}, M \text { and } A_{2}=\left\{C_{b}, Q\right\}\right. \text {. }
$$

Seller's unpredictability over the buyer's type can be expressed by the probability density function denoted by $g_{1}\left(\mu_{1}\right), \mu_{1} \in \theta_{1}$. The expected value is represented by $E_{\mu_{1}}$.

\section{The non-cooperative Stackelberg games}

Non-Cooperative game theory is very useful in modelling and understanding multi economic problems characterized by strategic interdependency. 
The non-cooperative Stackelberg strategic game structure was given in 1934 by Heinrich Stackelberg who published Market Structure and Equilibrium which illustrated the model and was a breaking point in the study of market structure. This is used to form the association between the partners of the supplychain. In this model, two players, seller and buyer interact with each other. One player performs as the leader and take the first move initiative and another player acts as the follower, move sequentially and shows his best response based on given information. The aim of the leader is to create the best policies based on the best response given by the buyer to maximize his profit.

\subsection{The seller-Stackelberg model with asymmetric information structure}

In this model, buyer behaves as the follower while the seller behaves as the leader. Seller moves first and offers selling price, $c_{b}$, and order quantity, $Q$ to the buyer in the situation under which demand is unknown to seller. The buyer chooses his best response according to the seller policy with the aim to increase his expected total profit. Based on the buyer's best move, the seller chooses his best strategy for finding the optimal selling price and lot size to gain his maximum profit.

$$
\begin{aligned}
\left.\operatorname{Max} E_{\mu_{1}} T P^{c}{ }_{s}\left(c_{b}, Q\right)\right) & =\int_{\mu_{1}}\left(\frac{D}{Q}\left(c_{b} Q-C Q-A_{s}\right)-\frac{1}{2} \operatorname{ICQ} \frac{D}{r}\right) g_{1}\left(\mu_{1}\right) d \mu_{1} \\
= & \int_{\mu_{1}}\left(\frac{1}{Q} K p_{b}{ }^{-e} M^{\beta}\left(c_{b} Q-C Q-A_{s}\right)-\frac{1}{2} I C Q u^{-1}\right) g_{1}\left(\mu_{1}\right) d \mu_{1}
\end{aligned}
$$

subject to

$$
\begin{aligned}
& p_{b}=\frac{e}{(e-\beta-1)(1-E[\alpha])}\left[c_{b}+\frac{A_{b}}{Q}+I c_{b} E[\alpha] Q-c_{s} E[\alpha]\right], \beta+1<\mathrm{e} \\
& M=\frac{\beta}{(e-\beta-1)}\left[c_{b}+\frac{A_{b}}{Q}+I c_{b} E[\alpha] Q-c_{S} E[\alpha]\right], \beta+1<\mathrm{e}
\end{aligned}
$$

Cycle length, $T=\max \left(T_{1}, T_{2}\right)$

Substituting the Eq. (14) and Eq. (15) into Eq. (13), the problem is converted into an unconstrained non-linear function with two variables $c_{b}$ and $Q$, the optimal solution can be obtained by any software tool.

\subsection{The seller-Stackelberg model with symmetric information structure}

Under symmetric information structure, buyer and seller know about each other's activity carried out i.e. seller knows the market demand thus, element of randomness is ignored i.e. expectation operator can be omitted. Now, seller Stackelberg problem becomes

$$
\begin{aligned}
\operatorname{Max} E_{\mu_{1}}\left(\mathrm{TP}_{s}^{c}\left(c_{b}, Q\right)\right)=\frac{D}{Q}\left(c_{b} Q-C Q-A_{s}\right)-\frac{1}{2} \operatorname{ICQ} \frac{D}{r} \\
=\frac{1}{Q} K p_{b}{ }^{-e} M^{\beta}\left(c_{b} Q-C Q-A_{s}\right)-\frac{1}{2} I C Q u^{-1}
\end{aligned}
$$

subject to 


$$
\begin{aligned}
& p_{b}=\frac{e}{(e-\beta-1)(1-E[\alpha])}\left[c_{b}+\frac{A_{b}}{Q}+I c_{b} E[\alpha] Q-c_{s} E[\alpha]\right], \beta+1<e \\
& M=\frac{\beta}{(e-\beta-1)}\left[c_{b}+\frac{A_{b}}{Q}+I c_{b} E[\alpha] Q-c_{s} E[\alpha]\right], \beta+1<e
\end{aligned}
$$

Cycle length, $T=\max \left(T_{1}, T_{2}\right)$

Substituting the Eq. (14) and Eq. (15) into Eq. (13), the problem is converted into an unconstrained non-linear function with two variables $c_{b}$ and $Q$, the optimal solution can be obtained by any software tool.

\section{Numerical examples}

This example shows the effect of the defective items in seller Stackelberg game model under asymmetric information structure. Input parameters in this example are taken from two papers Esmaeili and Zeephongsekul (2010) and Jaggi et al. (2013) which are given below. Suppose, $A_{b}=\$ 40, A_{s}=$ $\$ 140, C=\$ 1.5, \mathrm{I}=0.1, \mathrm{u}=1.01, \mathrm{k}=3500, \mathrm{~F}=1.25, \mathrm{~L}=0.09$ and mean value $\overline{\mathrm{e}}=1.7$ standard deviation $\sigma_{\mathrm{e}}=0.01$. The fraction of imperfect quality item, $\alpha$, is uniformly distributed on $(a, b), 0<$ $a<b<1$ i. e.,$\alpha \sim \mathrm{U}(\mathrm{a}, \mathrm{b})$.With $\alpha \sim \mathrm{U}(\mathrm{a}, \mathrm{b}), \mathrm{E}[\alpha]=\frac{\mathrm{a}+\mathrm{b}}{2}$ and $E\left[(1-\alpha)^{2}\right]$ which can be determined with formula $E\left[(1-\alpha)^{2}\right]=\int_{a}^{b}(1-\alpha)^{2} f(\alpha) d \alpha=\frac{a^{2}+a b+b^{2}}{3}+1-a-b$. The expected value of $\alpha$ is $E[\alpha]=0.02, E\left[(1-\alpha)^{2}\right]=0.960$, where $a=0$ and $b=0.04, \lambda=175200$ unit $/$ year, $c_{s}=\$ 5$.

\section{Example 1}

An example is shown to show the effect of the defective items in the seller Stackelberg game model under symmetric information structure. Seller Stackelberg model gives the following optimal values, Eq. (16) gives the results, $Q^{*}=249$ units and $c_{b}{ }^{*}=\$ 5.92$. Eq. (17) and Eq. (18) yield the results, $p_{b}{ }^{*}=\$ 18.88, M^{*}=\$ 1.63$. With these results, the seller's profit, $E\left[\operatorname{TP}_{s}^{c}\right]^{*}=\$ 80.08$ and the buyer's expected profit, $E\left[\mathrm{TP}^{c}{ }_{b}^{*}=\$ 211.11\right.$

\section{Example 2}

An example is shown to show the effect of the defective items in the seller Stackelberg game model under asymmetric information structure. Seller Stackelberg model gives the following optimal values. Eq. (13) gives the results, $Q^{*}=247$ units and $c_{b}{ }^{*}=\$ 5.96$. Eq. (14) and Eq. (15) yield the results, $p_{b}{ }^{*}=\$ 18.987$ and $M^{*}=\$ 1.63$, with these results, the seller's profit, $E\left[\mathrm{TP}^{c}{ }_{s}\right]^{*}=\$ 79.69$ and the buyer's expected profit, $E\left[\mathrm{TP}^{c}{ }_{b}\right]^{*}=\$ 210.37$.

\section{Sensitivity analysis}

Sensitivity analysis is performed to investigate the impact of parameters $\alpha, K$ on the different parameters $c_{b}, p_{b}, M, D, Q, E\left[\mathrm{TP}_{s}^{c}\right], E\left[\mathrm{TP}_{b}^{c}\right]$ in the non-cooperative seller Stackelberg game model by taking both the information structure: symmetric and asymmetric. Results of sensitivity analysis are shown through the Tables (1-4).

\section{Table 1}

Seller Stackelberg's sensitivity analysis with respect to $\alpha$ in symmetric information structure

\begin{tabular}{llllllll}
\hline \multicolumn{1}{c}{$\boldsymbol{\alpha}^{*}$} & \multicolumn{1}{c}{$\boldsymbol{c}_{\boldsymbol{b}}{ }^{*}$} & $\boldsymbol{Q}^{*}$ & \multicolumn{1}{c}{$\boldsymbol{p}_{\boldsymbol{b}}{ }^{*}$} & \multicolumn{1}{c}{$\boldsymbol{M}^{*}$} & $\boldsymbol{D}^{*}$ & $\boldsymbol{E}\left[\mathbf{T P}^{\boldsymbol{c}}{ }_{\boldsymbol{b}}\right]^{*}$ & $\boldsymbol{E}^{*} \mathbf{T P}^{\boldsymbol{c}}{ }_{\boldsymbol{s}}{ }^{*}$ \\
\hline 0.005 & 6.10 & 244 & 19.37 & 1.70 & 24 & 206.05 & 80.75 \\
0.010 & 6.04 & 246 & 19.21 & 1.68 & 25 & 207.52 & 80.52 \\
0.015 & 5.982 & 247 & 19.044 & 1.655 & 25 & 209.46 & 80.30 \\
0.020 & 5.92 & 249 & 18.88 & 1.63 & 25 & 211.11 & 80.08 \\
\hline
\end{tabular}




\begin{tabular}{llllllll}
\hline 0.025 & 5.87 & 250 & 18.707 & 1.61 & 26 & 213.08 & 79.86 \\
\hline
\end{tabular}

Table 2

Seller Stackelberg's sensitivity analysis with respect to $\alpha$ in asymmetric information structure

\begin{tabular}{|c|c|c|c|c|c|c|c|}
\hline $\boldsymbol{\alpha}^{*}$ & $\boldsymbol{c}_{\boldsymbol{b}}{ }^{*}$ & $\boldsymbol{Q}^{*}$ & $\boldsymbol{p}_{\boldsymbol{b}}{ }^{*}$ & $\boldsymbol{M}^{*}$ & $D^{*}$ & $E\left[\mathbf{T P}_{b}^{c}\right]^{*}$ & $\boldsymbol{E}\left[\mathbf{T P}_{s}^{c}\right]^{*}$ \\
\hline 0.005 & 6.13 & 243 & 19.48 & 1.71 & 24 & 205.05 & 80.37 \\
\hline 0.010 & 6.07 & 244 & 19.32 & 1.69 & 25 & 206.87 & 80.14 \\
\hline 0.015 & 6.02 & 246 & 19.15 & 1.66 & 25 & 208.46 & 79.91 \\
\hline 0.020 & 5.96 & 247 & 18.99 & 1.64 & 25 & 210.37 & 79.69 \\
\hline 0.025 & 5.90 & 249 & 18.82 & 1.62 & 26 & 212.04 & 79.47 \\
\hline
\end{tabular}

Table 3

Seller Stackelberg's sensitivity analysis with respect to k in asymmetric information structure

\begin{tabular}{llllllll}
\hline$k^{*}$ & $\boldsymbol{c}_{\boldsymbol{b}}{ }^{*}$ & $\boldsymbol{Q}^{*}$ & $\boldsymbol{p}_{\boldsymbol{b}}{ }^{*}$ & $\boldsymbol{M}^{*}$ & $\boldsymbol{D}^{*}$ & $\boldsymbol{E}\left[\mathbf{T P}^{\boldsymbol{c}}{ }_{\boldsymbol{b}}\right]^{*}$ & $\left.\boldsymbol{E}_{\left[\mathbf{T P}^{\boldsymbol{c}}\right.}{ }_{\boldsymbol{s}}\right]^{*}$ \\
\hline 2500 & 6.46 & 195 & 20.72 & 1.79 & 16 & 130.57 & 52.27 \\
3000 & 6.17 & 222 & 19.72 & 1.71 & 20 & 170.03 & 65.82 \\
3500 & 5.96 & 247 & 18.99 & 1.64 & 25 & 210.33 & 79.69 \\
4000 & 5.80 & 270 & 18.43 & 1.59 & 30 & 251.50 & 93.81 \\
4500 & 5.67 & 292 & 17.98 & 1.56 & 35 & 293.14 & 108.15 \\
\hline
\end{tabular}

Table 4

Seller Stackelberg's sensitivity analysis with respect to k in symmetric information structure

\begin{tabular}{lllccccc}
\hline$k^{*}$ & $\boldsymbol{c}_{\boldsymbol{b}}{ }^{*}$ & $\boldsymbol{Q}^{*}$ & $\boldsymbol{p}_{\boldsymbol{b}}{ }^{*}$ & $\boldsymbol{M}^{*}$ & $\boldsymbol{D}^{*}$ & $\left.\boldsymbol{E}\left[\mathbf{T P}^{\boldsymbol{c}}{ }_{\boldsymbol{b}}\right]^{*} \boldsymbol{E}^{*} \mathbf{T P}^{\boldsymbol{c}}\right]_{\boldsymbol{s}}^{*}$ \\
\hline 2500 & 6.42 & 197 & 20.58 & 1.78 & 16 & 131.01 & 52.52 \\
3000 & 6.13 & 224 & 19.59 & 1.69 & 21 & 170.65 & 66.13 \\
3500 & 5.92 & 249 & 18.88 & 1.63 & 26 & 211.11 & 80.08 \\
4000 & 5.76 & 272 & 18.33 & 1.59 & 31 & 252.39 & 94.28 \\
4500 & 5.63 & 294 & 17.88 & 1.55 & 36 & 294.18 & 108.69 \\
\hline
\end{tabular}

\section{Observations}

In a symmetric information structure, it is reflected from the Table 1 that as the percentage of imperfect quantity items goes up, the optimal selling price of the buyer, selling price of the seller and marketing expenditure go down, but optimal order quantity increases. Since the demand and order quantity increase, the buyer's profit increases. In an asymmetric information structure, it is depicted from the Table 2 that as the percentage of imperfect quantity items increases, then the demand of the quantity and order quantity increase, which results in more profit to the buyer. We can also observe that the profit of both the players in asymmetric information structure is lesser as compared to symmetric information structure. Thus, it is advised for both the players that they should have cordial relation between them and information sharing environment for good business.

In both the symmetric and asymmetric information structure, it is reflected from the Tables 3 and 4 that when the value of $K$ goes up, the selling price decreases marginally while the lot size increases and the present worth of profit of buyer and seller increases significantly. Further, it is also observed from Tables 3 and 4 that demand is increasing as $k$ increases but profit is lesser in asymmetric information structure as compared to symmetric information structure.

\section{Conclusions}

This study considered supply chain model for imperfect quality items with asymmetric and symmetric information structure and interaction of two players is modeled by seller Stackelberg approach. In this paper, it is presumed that the demand function depends on the selling price of the buyer and marketing expenditure. Optimal solutions are obtained in this approach. Effect of defective items and scaling constant demand function on decision variables of both the players and their profits are shown through 
sensitivity analysis in both the symmetric and asymmetric structure. Numerical examples show that in symmetric information structure, the buyer's and seller's profits are more than those obtained in the asymmetric structure. When demand function is not known to the seller, then seller's profit is lesser, even though seller is superior the buyer. Thus, both the players will be benefitted in case of complete and cordial information structure. This present model can be extended by taking the shortage cost, which is not considered in this paper. In this model, we have considered that defective items are sold at a discounted price. Instead of that, return policy can be applied, in this case transportation cost will be added.

\section{References}

Abad, P. L. (1994). Supplier pricing and lot sizing when demand is price sensitive. European Journal of Operational Research, 78(3), 334-354.

Abad, P. L. \& Jaggi, C.K. (2003).Joint approach for setting unit price and the length of the credit period for a seller when end demand is price sensitive. International Journal of Production Economics, 83(2), 115-122.

Bazaraa, M. S., Sherali, H. D., \& Shetty, C. M. (2013). Nonlinear programming: theory and algorithms. John Wiley \& Sons.

Cárdenas-Barrón, L.E. (2000) Observation on: economic production quantity model for items with imperfect quality. International journal of Production Economics, 67(2), 201.

Chan, C. K., \& Kingsman, B. G. (2007). Coordination in a single-vendor multi-buyer supply chain by synchronizing delivery and production cycles. Transportation Research Part E: Logistics and Transportation Review, 43(2), 90-111.

Chiang, C.W., Fitzsimmons, J., Huang, Z. \& Li, Susan X. (1994). A game theoretic approach to quantity discount problem. Decision Sciences, 25(1),153-168.

Corbett, C.J., \& de Groote, X. (2000). A supplier's optimal quantity discount policy under asymmetric information. Management Science, 46(3), 444-450

Dai, T., \& Qi, X. (2007). An acquisition policy for a multi-supplier system with a finite-time horizon. Computers \& operations research, 34(9), 2758-2773.

Esmaeili, M., Aryanezhad, M. B., \& Zeephongsekul, P. (2009). A game theory approach in sellerbuyer supply chain. European Journal of Operational Research, 195(2), 442-448.

Esmaeili, M., \& Zeephongsekul, P. (2010). Seller-buyer models of supply chain management with an asymmetric information structure. International Journal of Production Economics, 123(1), 146154.

Freeland, J. R. (1980). Coordination strategies for production and marketing in a functionally decentralized firm. AIIE Transactions, 12(2), 126-132.

Cachon, G. P., \& Lariviere, M. A. (2005). Supply chain coordination with revenue-sharing contracts: strengths and limitations. Management science, 51(1), 30-44.

Goyal, S. K., \& Cárdenas-Barrón, L. E. (2002). Note on: economic production quantity model for items with imperfect quality-a practical approach. International Journal of Production Economics, 77(1), 85-87.

Harsanyi, J. (1967) Games with incomplete information played by 'Bayesian' players. Part I.The basic model. Management Science, 14(3), 159-189.

Harsanyi, J. (1968a) Games with incomplete information played by 'Bayesian' players. Part II. Bayesian equilibrium points. Management Science, 14(5), 320-334

Harsanyi, J. (1968b) Games with incomplete information played by'Bayesian' players. Part III. The basic probability distribution of the game. Management Science, 14(7), 486-502.

Van den Heuvel, W., Borm, P., \& Hamers, H. (2007). Economic lot-sizing games. European Journal of Operational Research, 176(2), 1117-1130.

Jaggi, C.K., Goel, S.K., \& Mittal, M. (2013). Credit financing in economic ordering policies for defective items with allowable shortages. Applied Mathematics and Computation, 219(10), 52685282. 
Jung, H., \& Klein, C. M. (2005). Optimal inventory policies for an economic order quantity model with decreasing cost functions. European Journal of Operational Research, 165(1), 108-126.

Jung, H., \& Klein, C. M. (2001). Optimal inventory policies under decreasing cost functions via geometric programming. European Journal of Operational Research, 132(3), 628-642.

Lau, A. H. L., \& Lau, H. S. (2005). Some two-echelon supply-chain games: Improving from deterministic-symmetric-information to stochastic-asymmetric-information models. European Journal of Operational Research, 161(1), 203-223.

Lau, A. H. L., Lau, H. S., \& Zhou, Y. W. (2007). A stochastic and asymmetric-information framework for a dominant-manufacturer supply chain. European Journal of Operational Research, 176(1), 295-316.

Lee, W. J. (1993). Determining order quantity and selling price by geometric programming: optimal solution, bounds, and sensitivity. Decision Sciences, 24(1), 76-87.

Lee, W. J., \& Kim, D. (1993). Optimal and heuristic decision strategies for integrated production and marketing planning. Decision Sciences, 24(6), 1203-1214.

Kim, D., \& Lee, W. J. (1998). Optimal coordination strategies for production and marketing decisions. Operations Research Letters, 22(1), 41-47.

Lee, W. J., Kim, D., \& Cabot, A. V. (1996). Optimal demand rate, lot sizing, and process reliability improvement decisions. IIE transactions, 28(11), 941-952.

Maddah, B., \& Jaber, M. Y. (2008). Economic order quantity for items with imperfect quality: revisited. International Journal of Production Economics, 112(2), 808-815.

Papachristos, S., \& Konstantaras, I. (2006). Economic ordering quantity models for items with imperfect quality. International Journal of Production Economics, 100(1), 148-154.

Reyes, P. M. (2005). A mathematical example of the two-echelon inventory model with asymmetric market information. Applied Mathematics and Computation, 162(1), 257-264.

Sadjadi, S. J., Oroujee, M., \& Aryanezhad, M. B. (2005). Optimal production and marketing planning. Computational Optimization and Applications, 30(2), 195-203.

Salameh, M. K., \& Jaber, M. Y. (2000). Economic production quantity model for items with imperfect quality. International journal of production economics, 64(1), 59-64.

Sarmah, S. P., Acharya, D., \& Goyal, S. K. (2006). Buyer vendor coordination models in supply chain management. European journal of operational research, 175(1), 1-15.

Schwaller, R. L. (1988). EOQ under inspection costs. Production and Inventory Management Journal, 29(3), 22.

Sucky, E. (2005). Inventory management in supply chains: A bargaining problem. International Journal of Production Economics, 93, 253-262.

Sucky, E. (2006). A bargaining model with asymmetric information for a single supplier-single buyer problem. European Journal of Operational Research, 171(2), 516-535.

Weng, Z. K. (1995). Channel coordination and quantity discounts. Management science, 41(9), 15091522.

Zhang, X., \& Zeephongsekul, P. (2013). Asymmetric information supply chain models with credit option. Industrial Engineering and Management Systems, 12(3), 264-273.

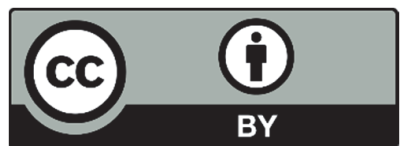

(C) 2018 by the authors; licensee Growing Science, Canada. This is an open access article distributed under the terms and conditions of the Creative Commons Attribution (CC-BY) license (http://creativecommons.org/licenses/by/4.0/). 